\title{
Frecuencia de factores de riesgo cardiovascular en pacientes con síndrome de apnea obstructiva del sueño
}

\section{Frequency of cardiovascular risk factors in patients with obstructive sleep apnea syndrome}

\author{
Ana Gallmann ${ }^{1}$, Fernando Camporro ${ }^{1}$, Florencia Gazzoni ${ }^{1}$. \\ 1.Universidad católica de Córdoba. Clínica Universitaria Reina Fabiola. Servicio de Clínica Médica. \\ Correspondencia: Gallmann, Ana. Servicio de Clínica Médica. Clínica Universitaria Reina Fabiola Oncativo 1248-X5004FHP-Cordoba, Argentina; email: \\ anagallmann@gmail.com
}

\section{Resumen}

INTRODUCCIÓN: La apnea obstructiva del sueño afecta al 14\% de los hombres y al 5\% de las mujeres. Diversos estudios han demostrado la asociación entre el síndrome de apnea obstructiva del sueño (SAOS) y enfermedades cardiovasculares. Los pacientes con SAOS tienen aproximadamente cinco veces más riesgo de desarrollar enfermedad cardiovascular a siete años.

OBJETIVO: Estimar la frecuencia de factores de riesgo cardiovascular en una muestra de pacientes con Apnea Obstructiva del Sueño (AOS).

PACIENTES Y MÉTODOS Se realizó un estudio observacional, transversal, descriptivo. Se incluyeron pacientes consecutivos mayores de 18 años diagnosticados con SAOS en el laboratorio de sueño de la Clínica Universitaria Reina Fabiola, durante el periodo comprendido entre el entre el 1 de junio de 2018 y 30 de diciembre de 2018. Se registraron las siguientes variables: edad, sexo, índice de masa corporal (IMC), hipertensión arterial (HTA), hábito tabáquico, diabetes, dislipemia, enfermedad cardiovascular aterosclerótica clínica y evaluación del riesgo cardiovascular (RCV) a 10 años calculado a través del Cardiovascular Risk Calculator PAHO/WHO. Se realizó una descripción de las variables cuantitativas mediante medidas de posición y dispersión. Las variables cualitativas se expresaron en porcentajes.

RESULTADOS: Se incluyeron 99 pacientes. El 79,8\% fueron hombres, con una media (desviación estándar, DE) de edad de 44,84 $\pm 13,55$ años y el 20,20\% fueron mujeres, con una media (DE) de edad de $46,15 \pm 11,54$ años.

El principal factor de riesgo cardiovascular (RCV) encontrado fue dislipemia $(77,78 \%)$, seguido de HTA $(38,38 \%)$ y obesidad $(56,56 \%)$ o sobrepeso $(34,34 \%)$. El $64,27 \%$ de los pacientes con AOS leve, el $84 \%$ de los pacientes con AOS moderada y el $95 \%$ de los pacientes con AOS grave, tuvieron dos o más factores de RCV.

$\mathrm{Al}$ calcular el RCV global a 10 años, el 91,92\% de los pacientes tuvieron un RCV bajo, el 2,02\% moderado y el 6,06\% muy alto. Ningún paciente tuvo RCV alto. El $87,88 \%$ de los pacientes tenían dos o más factores de RCV

CONCLUSIONES: Destacamos la relación, ya descrita en la literatura, entre los factores de RCV y la AOS. La misma fue más notoria al observar la cantidad de pacientes que además de AOS presentaron HTA, diabetes y obesidad, condiciones altamente prevalentes en nuestra sociedad. La mayoría de los pacientes con AOS presentó dos o más factores de RCV.

Palabras claves: Síndrome de apnea obstructiva del sueño. Riesgo cardiovascular. 


\begin{abstract}
INTRODUCTION: The frequency of obstructive sleep apnea is 14\% in men and 5\% in women. Several studies have shown the association between obstructive sleep apnea syndrome (OSAS) and cardiovascular diseases. Patients with OSAS have approximately five times the risk of developing cardiovascular disease at seven years.

OBJECTIVE: To estimate de frequency of cardiovascular risk factors in a sample of patients with OSAS. PATIENTS AND METHODS: We performed an observational, cross-sectional, descriptive study. We included consecutive patients over 18 years of age diagnosed with OSA during the period between June 1, 2018 and December 30, 2018. The following variables were recorded: age, sex, body mass index (BMI), arterial hypertensión (AHT), smoking, diabetes, dyslipidemia, clinical atherosclerotic cardiovascular disease and 10-year cardiovascular risk assessment (CVR) calculated through the Cardiovascular Risk PAHO / WHO calculator. We used descriptive statistics.

RESULTS: We included 99 patientes, fo them, $79.8 \%$ were men, with a mean (standard deviation, SD) age of $44.84 \pm 13.55$ years and $20.20 \%$ were women, with a mean age of $46.15 \pm 11.54$ years.

The main cardiovascular risk factor (CVR) found was dyslipidemia (77.78\%), followed by AHT (38.38\%) and obesity $(56.56 \%)$ or overweight $(34.34 \%)$. Sixty-four pecent of patients with mild OSA, $84 \%$ of patients with moderate OSA and $95 \%$ of patients with severe OSA, had 2 or more CVR factors.

When calculating the global CVR at 10 years, $91.92 \%$ of the patients had a low CVR, 2.02\% moderate and $6.06 \%$ very high. No patient had high CVR. The majority of the patients $(87.88 \%)$ hadtwo or more CVR factors.

CONCLUSIONS: We highlight the high frequency, as already described in the literature, of CVR factors and AOS.

The most frequent CVR factors were hypertension, diabetes and obesity, highly prevalent conditions in our society. The majority of the patients showed 2 or more CVR factors.
\end{abstract}

Key words: Obstructive sleep apnea syndrome. Cardiovascular risk.

\title{
Introducción
}

La apnea obstructiva del sueño (AOS) afecta al $14 \%$ de los hombres y al $5 \%$ de las mujeres ${ }^{1}$

. En la última década, el conocimiento relacionado a esta entidad ha evolucionado de manera creciente, principalmente en su relación con la salud en general, y en particular, como factor de riesgo cardiovascular. De hecho, distintos estudios han demostrado su asociación con las enfermedades cardiovasculares ${ }^{2-5}$. Distintos trabajos relacionan esta patología de manera directa con la ateroesclerosis ${ }^{6}$, disfunción endotelial $^{7}$, hipertensión arterial $(\text { HTA })^{8}$ y aumento de la morbilidad y mortalidad cardiovascular ${ }^{4}$. De hecho, los pacientes con síndrome de apnea obstructiva del sueño (SAOS) tienen aproximadamente cinco veces más riesgo de desarrollar enfermedad cardiovascular a siete años ${ }^{9}$

Entre los mecanismos responsables de las consecuencias cardiovasculares de esta enfermedad se mencionan la alteración en los ciclos de hipoxia y re-oxigenación durante el sueño fragmentado, cuya consecuencia es la isquemia transitoria de $\operatorname{los}$ tejidos ${ }^{10}$. De forma secundaria, aumentan los radicales libres de oxígeno que actúan como mediadores de citoquinas pro-inflamatorias, generando un estado de inflamación sistémica ${ }^{11}$, una mayor activación del sistema simpático ${ }^{12}$ disfunción endotelial, desregulación metabólica ${ }^{13}$ y un estado de hipercoagulabilidad ${ }^{14}$.

Debido al alto grado de morbimortalidad que tienen las enfermedades cardiovasculares, es importante conocer el impacto cardio-metabólico que supone la AOS para poder identificarlo y tratarlo de manera oportuna y correcta.

\section{Objetivo}

Estimar la frecuencia de factores de riesgo cardiovascular en una muestra de pacientes con AOS.

\section{Pacientes y métodos}

Se realizó un estudio observacional, transversal y descriptivo. Se incluyeron pacientes con AOS diagnosticada en el laboratorio de sueño de la Clínica Universitaria Reina Fabiola, a través de una polisomnografía nocturna, mediante un dispositivo Philips Alice 6 LDe, de 31 canales, durante el periodo comprendido entre el 1 de junio de 2018 y 30 de diciembre de 2018. 


\section{Criterios de inclusión}

Pacientes mayores de 18 años con AOS diagnosticada en el laboratorio de sueño de la Clínica Universitaria Reina Fabiola que hayan firmado el consentimiento para realizar estudios de laboratorio y participar de dicho protocolo.

\section{Criterios de exclusión}

Pacientes menores de 18 años, pacientes que carecían de los datos requeridos o pacientes que hayan presentado la negativa de participar.

\section{Variables registradas}

- $\quad$ Edad

- Sexo

- Índice de masa corporal (IMC), el cual corresponde a la relación entre el peso expresado en kilos y el cuadrado de la altura, expresada en metros, y permite, según la $\mathrm{OMS}^{15}$, clasificar a la obesidad en:

- Normo Peso: IMC 18.5 - 24.9

- Sobrepeso: IMC 25 - 29.9

- Obesidad Grado I o moderada: IMC 30 - 34.9

- Obesidad Grado II o severa: IMC 35 - 39.9

- Obesidad Grado III o mórbida: IMC $\geq 40$

- Dislipemia, definida como una alteración en el perfil lipídico, tomando como valores de corte, según las guías para el tratamiento de la dislipemia en el adulto ATP-III ${ }^{16}$, los siguientes:

- Colesterol Total $\geq 200 \mathrm{mg} / \mathrm{dl}$

- HDL < $40 \mathrm{mg} / \mathrm{dl}$ en hombres y <50mg/dl en mujeres.

- LDL $\geq 130 \mathrm{mg} / \mathrm{dl}$

- Triglicéridos $\geq 150 \mathrm{mg} / \mathrm{dl}$

También se consideraron dislipémicos a aquellos pacientes que, teniendo un perfil lipídico dentro de parámetros normales, estaban bajo tratamiento con medicación hipolipemiante.

- Hábito tabáquico (paquetes/año). Se consideraron ex tabaquistas a aquellos pacientes que abandonaron el hábito hacía 12 meses o más.

- Diabetes mellitus, definida de acuerdo con los criterios de la Asociación Americana de Diabetes (ADA) y la Organización Mundial para la Salud $(\mathrm{OMS})^{17}$ :
- Glucemia plasmática al azar $\geq 200 \mathrm{mg} / \mathrm{dl}$ en presencia de síntomas clásicos de diabetes

- Glucemia plasmática en ayunas (GPA) $>126 \mathrm{mg} / \mathrm{dl}$.

- Valor de glucemia 2 horas post prueba de tolerancia oral a la glucosa (PTOG) $\geq 200 \mathrm{mg} / \mathrm{dl}$

- Hemoglobina glicosilada $(\mathrm{HbA} 1 \mathrm{c})>6,5 \%$

También se incluyeron dentro de este grupo a los pacientes que estaban en tratamiento con insulina o hipoglucemiantes orales.

- Hipertensión arterial definida como PAS $\geq 140 \mathrm{mmHg}$ o $\mathrm{TAD} \geq 90 \mathrm{mmHg}$ en al menos dos mediciones por consulta en dos consultas distintas, según la Guía de Práctica Clínica de la ESH/ESC ${ }^{18}$ o por la toma de medicamentos antihipertensivos.

- Enfermedad cardiovascular aterosclerótica clínica que incluyó, según el $\mathrm{ACC}_{\mathrm{AHA}}{ }^{19}$, síndrome coronario agudo, antecedentes de infarto agudo de miocardio (IAM), angina estable o inestable, revascularización arterial coronaria o de otro tipo, accidente cerebrovascular (ACV), accidente isquémico transitorio (AIT) o enfermedad arterial periférica atribuida a un origen ateroesclerótico.

- Se calculó el riesgo cardiovascular (RCV) a 10 años (expresado en porcentaje y definido como el riesgo de padecer un evento cardiovascular fatal o no fatal a 10 años), usando las tablas de predicción de riesgo de la OMS mediante la aplicación WHO/PAHO Cardiovascular Risk Calculator (www.paho.org/cardioapp/web/20.

- Se consideró que un paciente posee comorbilidad cardiovascular ante un aumento del RCV a 10 años o la presencia de variables independientes: sobrepeso u obesidad, dislipemia, diabetes, HTA y enfermedad cardiovascular aterosclerótica clínica.

- Relacionadas a la PSG: La misma se realizó mediante un dispositivo Philips Alice 6 LDe, de 31 canales. Se valoró la cantidad de tiempo que el paciente desaturó por debajo de $90 \%$ (T90) y la saturación mínima alcanzada. Se midió el IAH. La gravedad del síndrome se clasificó de acuerdo con McNicholas, de la siguiente manera ${ }^{21}$ :

- Leve: $\mathrm{IAH} \geq 5 \leq 14,9$.

- Moderado: IAH $\geq 15 \leq 29,9$.

- Severo: $\mathrm{IAH} \geq 30$. 
Los datos fueron recolectados en una hoja de recolección de datos diseñada para tal fin (Apéndice II).

\section{Análisis estadístico}

Las características generales y la prevalencia de cada factor de riesgo se determinaron mediante estadística descriptiva. Se realizó una descripción de las variables cuantitativas mediante medidas de posición y dispersión. Las variables cualitativas se expresaron en porcentajes

\section{Resultados}

Se incluyó una serie consecutiva de 99 pacientes diagnosticados de AOS en el Laboratorio de Sueño de la Clínica Universitaria Reina Fabiola.

El 20,2\% ( $\mathrm{N}=20)$ de los pacientes fueron mujeres y el 79,8\% (N=79) fueron hombres, con una media (desviación estándar, DE) de edad de 46,15 $\pm 11,54$ años para las primeras y de $44,84 \pm 13,55$ años para los segundos.

La distribución de la gravedad de la AOS según sexo se detalla en la Tabla 1.

Tabla 1. Descripción de la severidad de la AOS en la población estudiada según sexo

\begin{tabular}{|c|c|c|c|c|c|c|}
\hline \multirow{2}{*}{$\begin{array}{l}\text { Apnea obstructiva del } \\
\text { sueño }\end{array}$} & \multicolumn{2}{|c|}{ Mujeres $(n=20)$} & \multicolumn{2}{|c|}{ Hombres $(n=79)$} & \multicolumn{2}{|c|}{ Total $(n=99)$} \\
\hline & $\mathrm{N}$ & $\%$ & $\mathrm{~N}$ & $\%$ & $\mathrm{~N}$ & $\%$ \\
\hline Leve & 7 & 35 & 7 & 8,86 & 14 & 14,14 \\
\hline Moderada & 5 & 25 & 20 & 25,32 & 25 & 25,25 \\
\hline Severa & 8 & 40 & 52 & 65,82 & 60 & 60,61 \\
\hline
\end{tabular}

El principal factor de RCV encontrado fue dislipemia, seguido de HTA y obesidad o sobrepeso. El

resto de las comorbilidades cardiovasculares se detallan en la Tabla 2.

Tabla 2. Descripción de los factores de riesgo cardiovascular en los pacientes con AOS.

\begin{tabular}{|c|c|c|c|c|c|c|c|}
\hline \multirow{2}{*}{\multicolumn{2}{|c|}{ Factores de riesgo cardiovascular }} & \multicolumn{2}{|c|}{ Mujeres } & \multicolumn{2}{|c|}{ Hombres } & \multicolumn{2}{|c|}{ Total } \\
\hline & & $\mathbf{N}$ & $\%$ & $\mathrm{~N}$ & $\%$ & $\mathbf{N}$ & $\%$ \\
\hline \multicolumn{2}{|c|}{ Hipertensión arterial } & 11 & 55 & 27 & 34,18 & 38 & 38,38 \\
\hline \multicolumn{2}{|l|}{ Dislipemia } & 16 & 80 & 61 & 77,22 & 77 & 77,78 \\
\hline \multicolumn{2}{|l|}{ Diabetes } & 2 & 10 & 13 & 16,46 & 15 & 15,15 \\
\hline \multicolumn{2}{|c|}{ Infarto agudo de miocardio } & 0 & 0 & 3 & 3,8 & 3 & 3,03 \\
\hline \multicolumn{2}{|c|}{ Revascularización } & 0 & 0 & 4 & 5,06 & 4 & 4,04 \\
\hline \multicolumn{2}{|c|}{ Accidente isquémico transitorio } & 0 & 0 & 0 & 0 & 0 & 0 \\
\hline \multicolumn{2}{|c|}{ Accidente cerebro vascular } & 0 & 0 & 2 & 2,53 & 2 & 2,02 \\
\hline \multicolumn{2}{|c|}{ Enfermedad Vascular periférica } & 0 & 0 & 3 & 3,8 & 3 & 3,03 \\
\hline \multicolumn{2}{|c|}{ Insuficiencia renal crónica } & 0 & 0 & 1 & 1,27 & 1 & 1,01 \\
\hline \multirow{4}{*}{ Obesidad } & Sobrepeso & 5 & 25 & 29 & 36,71 & 34 & 34,34 \\
\hline & Obesidad Grado I & 2 & 10 & 26 & 32,91 & 28 & 28,28 \\
\hline & Obesidad Grado II & 4 & 20 & 11 & 13,92 & 15 & 15,15 \\
\hline & Obesidad Grado III & 5 & 25 & 8 & 10,13 & 13 & 13,13 \\
\hline \multirow{2}{*}{ Tabaquismo } & sı & 3 & 15 & 19 & 24,05 & 22 & 22,22 \\
\hline & EX & 2 & 10 & 22 & 27,85 & 24 & 24,24 \\
\hline
\end{tabular}

Al analizar los factores de RCV como variables independientes se vio que el $87,88 \%$ de los pacientes tenían dos o más. Sin embargo, a pesar de la elevada frecuencia de factores de RCV, al calcular el RCV global a 10 años sólo el 6,06\% tuvo un RCV muy alto y ningún paciente tuvo un RCV alto.

En la Tabla 3 se detalla el RCV global a 10 años según la severidad de la AOS. A su vez, teniendo en cuenta la cantidad de factores de RCV que cada paciente presentó, pudimos observar que el $64,27 \%$ de los pacientes con AOS leve, el 84\% de los pacientes con AOS moderada y el $95 \%$ de los pacientes con AOS grave, tuvieron 2 o más factores de RCV Tabla 4.

Tabla 3. Descripción del riesgo cardiovascular según la severidad de la AOS.

\begin{tabular}{|c|c|c|c|c|c|c|c|}
\hline \multirow{2}{*}{\begin{tabular}{|c}
$\begin{array}{c}\text { Apnea } \\
\text { obstructiva } \\
\text { del sueño }\end{array}$ \\
\end{tabular}} & \multirow{2}{*}{$\begin{array}{c}\text { Riesgo } \\
\text { cardiovascular }\end{array}$} & \multicolumn{2}{|c|}{ Mujeres ( $n=20$ ) } & \multicolumn{2}{|c|}{ Hombres $(n=79)$} & \multicolumn{2}{|c|}{ Total $(n=99)$} \\
\hline & & $\mathrm{N}$ & $\%$ & $\mathrm{~N}$ & $\%$ & $\mathbf{N}$ & $\%$ \\
\hline \multirow{4}{*}{ Leve } & Bajo & 7 & 100 & 6 & 8,71 & 13 & 92,86 \\
\hline & Moderado & 0 & 0 & 0 & 0 & 0 & 0 \\
\hline & Alto & 0 & 0 & 0 & 0 & 0 & 0 \\
\hline & Muy Alto & 0 & 0 & 1 & 14,29 & 1 & 7,14 \\
\hline \multirow{4}{*}{ Moderada } & Bajo & 5 & 100 & 17 & 85 & 22 & 88 \\
\hline & Moderado & 0 & 0 & 0 & 0 & 0 & 0 \\
\hline & Alto & 0 & 0 & 0 & 0 & 0 & 0 \\
\hline & Muy Alto & 0 & 0 & 3 & 15 & 3 & 12 \\
\hline \multirow{4}{*}{ Severo } & Bajo & 8 & 100 & 48 & 92,31 & 56 & 92,33 \\
\hline & Moderado & 0 & 0 & 2 & 3,85 & 2 & 3,33 \\
\hline & Alto & 0 & 0 & 0 & 0 & 0 & 0 \\
\hline & Muy Alto & 0 & 0 & 2 & 3,85 & 2 & 3,33 \\
\hline
\end{tabular}

Tabla 4. Descripción del número de factores de riesgo cardiovascular según la severidad de la AOS.

\begin{tabular}{|c|c|c|c|c|c|c|c|}
\hline \multirow{2}{*}{$\begin{array}{c}\text { Apnea } \\
\text { obstructiva } \\
\text { del sueño }\end{array}$} & \multirow{2}{*}{$\begin{array}{c}\text { Factores de } \\
\text { riesgo } \\
\text { cardiovascular }\end{array}$} & \multicolumn{2}{|c|}{ Mujeres ( $n=20)$} & \multicolumn{2}{|c|}{ Hombres $(n=79)$} & \multicolumn{2}{|c|}{ Total $(n=99)$} \\
\hline & & $\mathrm{N}$ & $\%$ & $\mathrm{~N}$ & $\%$ & $\mathrm{~N}$ & $\%$ \\
\hline \multirow{7}{*}{ Leve } & 0 & 1 & 14,29 & 1 & 14,29 & 2 & 14,29 \\
\hline & 1 & 2 & 28,57 & 1 & 14,29 & 3 & 21,43 \\
\hline & 2 & 2 & 28,57 & 3 & 42,86 & 5 & 35,7 \\
\hline & 3 & 1 & 14,29 & 1 & 14,29 & 2 & 14,29 \\
\hline & 4 & 1 & 14,29 & 0 & 0 & 1 & 7,14 \\
\hline & 5 & 0 & 0 & 0 & 0 & 0 & 0 \\
\hline & 8 & 0 & 0 & 1 & 14,29 & 1 & 7,14 \\
\hline \multirow{7}{*}{ Moderada } & 0 & 0 & 0 & 2 & 10 & 2 & 8 \\
\hline & 1 & 0 & 0 & 2 & 10 & 2 & 8 \\
\hline & 2 & 2 & 40 & 6 & 30 & 8 & 32 \\
\hline & 3 & 2 & 40 & 5 & 25 & 7 & 28 \\
\hline & 4 & 1 & 20 & 3 & 15 & 4 & 16 \\
\hline & 5 & 0 & 0 & 2 & 10 & 2 & 8 \\
\hline & 8 & 0 & 0 & 0 & 0 & 0 & 0 \\
\hline \multirow{7}{*}{ Severo } & 0 & 0 & 0 & 1 & 1,92 & 1 & 1,67 \\
\hline & 1 & 1 & 12,5 & 1 & 1,92 & 2 & 3,33 \\
\hline & 2 & 3 & 37,5 & 25 & 48,08 & 28 & 46,67 \\
\hline & 3 & 3 & 37,5 & 18 & 34,62 & 21 & 35,00 \\
\hline & 4 & 0 & 0 & 5 & 9,62 & 5 & 8,33 \\
\hline & 5 & 1 & 12,5 & 2 & 3,85 & 3 & 5,00 \\
\hline & 8 & 0 & 0 & 0 & 0 & 0 & 0,00 \\
\hline
\end{tabular}

En la Tabla 5 se desglosa la frecuencia de los factores de riesgo cardiovascular de acuerdo a la severidad de la apnea obstructiva del sueño.

Revista Methodo: Investigación Aplicada a las Ciencias Biológicas. Universidad Católica de Córdoba. Jacinto Ríos 571 Bo Gral. Paz. X5004FXS. Córdoba. Argentina. Tel.: (54) 3514517299 / Correo: methodo@ucc.edu.ar / Web: methodo.ucc.edu.ar | ARTICULO ORIGINAL Rev. Methodo 2020;5(2):56-62 
Tabla 5. Descripción de los FRCV según la everidad de la AOS.

\begin{tabular}{|c|c|c|c|c|c|c|c|}
\hline \multirow{2}{*}{$\begin{array}{l}\begin{array}{l}\text { Factor de riesgo } \\
\text { cardiovascular }\end{array} \\
\end{array}$} & \multirow{2}{*}{$\begin{array}{c}\text { Apnea } \\
\text { obstructiva del } \\
\text { sueño }\end{array}$} & \multicolumn{2}{|c|}{ Mujeres } & \multicolumn{2}{|c|}{ Hombres } & \multicolumn{2}{|c|}{ Total } \\
\hline & & $\mathrm{N}$ & $\%$ & $\mathbf{N}$ & $\%$ & $\mathrm{~N}$ & $\%$ \\
\hline \multirow{3}{*}{ Hipertensión arterial } & Leve & 3 & 42,86 & 1 & 14,29 & 4 & 28,27 \\
\hline & Moderada & 4 & 80 & 6 & 30 & 10 & 40 \\
\hline & Severa & 4 & 50 & 20 & 38,46 & 24 & 40 \\
\hline \multirow{3}{*}{ Dislipemia } & Leve & 5 & 71,43 & 6 & 85,71 & 11 & 78,57 \\
\hline & Moderada & 5 & 100 & 13 & 65 & 18 & 72 \\
\hline & Severa & 6 & 75 & 42 & 80,77 & 48 & 80 \\
\hline \multirow{3}{*}{ Diabetes } & Leve & 0 & 0 & 1 & 14,29 & 1 & 7,14 \\
\hline & Moderado & 1 & 20 & 3 & 15 & 4 & 16 \\
\hline & Severo & 1 & 12,5 & 9 & 17,31 & 10 & 16,67 \\
\hline \multirow{3}{*}{$\begin{array}{l}\text { Infarto agudo de } \\
\text { miocardio }\end{array}$} & Leve & 0 & 0 & 1 & 14,29 & 1 & 7,14 \\
\hline & Moderada & 0 & 0 & 1 & 5 & 1 & 4 \\
\hline & Severa & 0 & 0 & 1 & 1,92 & 1 & 1,67 \\
\hline \multirow{3}{*}{ Revascularización } & Leve & 0 & 0 & 1 & 14,29 & 1 & 7,14 \\
\hline & Moderada & 0 & 0 & 2 & 10 & 2 & 8 \\
\hline & Severa & 0 & 0 & 1 & 1,92 & 1 & 1,67 \\
\hline \multirow{3}{*}{$\begin{array}{l}\text { Accidente cerebro } \\
\text { vascular }\end{array}$} & Leve & 0 & 0 & 1 & 14,29 & 1 & 7,14 \\
\hline & Moderada & 0 & 0 & 1 & 5 & 1 & 4 \\
\hline & Severa & 0 & 0 & 0 & 0 & 0 & 0 \\
\hline \multirow{3}{*}{$\begin{array}{l}\text { Enfermedad Vascular } \\
\text { Periférica. }\end{array}$} & Leve & 0 & 0 & 1 & 14,29 & 1 & 7,14 \\
\hline & Moderada & 0 & 0 & 1 & 5 & 1 & 4 \\
\hline & Severa & 0 & 0 & 1 & 1,92 & 1 & 1,67 \\
\hline \multirow{3}{*}{$\begin{array}{l}\text { Insuficiencia renal } \\
\text { crónica }\end{array}$} & Leve & 0 & 0 & 0 & 0 & 0 & 0 \\
\hline & Moderado & 0 & 0 & 0 & 0 & 0 & 0 \\
\hline & Severo & 0 & 0 & 1 & 1,62 & 1 & 1,97 \\
\hline \multirow{3}{*}{ Tabaquismo } & Leve & 1 & 14,29 & 2 & 28,57 & 3 & 21,43 \\
\hline & Moderada & 0 & 0 & 7 & 35 & 7 & 28 \\
\hline & Severa & 2 & 15 & 10 & 19,23 & 12 & 20 \\
\hline
\end{tabular}

\section{Discusión}

Al analizar los datos obtenidos a partir de nuestra población con AOS, se destaca la frecuencia de presentación de comorbilidades cardiovasculares. Debemos destacar el alto porcentaje de pacientes que presentaron dos o más factores de RCV, siendo los más frecuentes la dislipemia, la obesidad y la HTA. A su vez pudimos apreciar un incremento de su frecuencia a medida que la severidad de la AOS aumentaba: en el grupo de pacientes con AOS leve el $64,29 \%$ presentó dos o más FRCV, aumentando al $84 \%$ en el grupo con AOS moderada y al $95 \%$ en los pacientes con AOS severa. Estos datos son coincidentes con la literatura, donde se describe dicha relación ${ }^{2-5,9,11,22 .}$ Sin embargo, a pesar de la elevada proporción de pacientes que presentaban dos o más factores de RCV, al calcular el RCV global, la mayoría tenían un RCV bajo, es decir, menor al $10 \%$ para padecer un evento cardiovascular fatal o no fatal en los próximos 10 años. Los resultados al respecto en la literatura son controvertidos. $\mathrm{Si}$ bien, como mencionamos previamente, esta relación está descrita en múltiples estudios, en 2012 se publicó un metaanálisis en el que se concluyó que la AOS parece estar asociada con $\mathrm{ACV}$, pero la relación con la cardiopatía isquémica y la mortalidad cardiovascular necesita más investigación23 y otra publicación actual plantea la duda sobre si la hipoxia crónica puede estimular el desarrollo de vasos colaterales cumpliendo un papel protector o si es un factor de riesgo para un peor pronóstico después de padecer un evento cardiovascular ${ }^{24}$. Además, podemos atribuir el bajo RCV calculado a 10 años al bajo promedio de edad de nuestra población. Al analizar cada factor de RCV de forma independiente, encontramos un aumento de la frecuencia, a medida que aumentaba la severidad de la AOS en los casos de HTA, diabetes e IRC; para las condiciones restantes, esta relación no fue manifiesta.

Pudimos observar que la minoría de los pacientes de nuestra población se encontraron dentro de los márgenes de un peso ideal y la mayoría presentaron alguna alteración en su perfil lipídico o estaban tratados para dislipemia. Además, fue notorio cómo, a medida que aumenta la severidad de la AOS, disminuye el porcentaje de pacientes con normo peso y aumenta el de pacientes con sobrepeso o algún grado de obesidad, datos coincidentes con la literatura ${ }^{2,10,25}$.

$\mathrm{Al}$ carecer de un grupo de pacientes control o de datos epidemiológicos que informen la frecuencia de factores de RCV en la población general, comparamos nuestros resultados con una muestra de pacientes estudiada en una revisión sistemática sobre estudios de Argentina entre enero de 1988 y diciembre de 2014 por el Grupo de Trabajo en Epidemiología de la Sociedad Argentina de Hipertensión Arterial y el CONICET $^{26}$, que probablemente refleje una aproximación a la epidemiología de nuestro país. En dicho estudio, el $38,8 \%$ de los pacientes fueron dislipémicos, el $33,4 \%$ hipertensos, el $32,1 \%$ obesos y el $7,5 \%$ diabéticos. Comparando los resultados con los de nuestro estudio, pudimos observar una gran diferencia en la frecuencia de factores de RCV, ya que los porcentajes en nuestros pacientes con AOS fueron notablemente más altos.

Consideramos que los pacientes con AOS deberían englobarse dentro de una población propensa a padecer comorbilidad cardiovascular. Por ende, tanto el clínico como cualquier especialista que sospeche y/o diagnostique esta condición debería conocer el impacto cardio-metabólico que la misma supone, para poder identificarlo y tratarlo de manera oportuna y adecuada, con un enfoque multidisciplinario. Por lo tanto, es necesario contemplar, para su manejo, la necesidad de realizar un estudio exhaustivo en busca de otras patologías asociadas y un seguimiento idóneo para detectar oportunamente el desarrollo de las mismas. A la inversa, ante pacientes que padezcan alguna comorbilidad cardiovascular, se debería sospechar y buscar datos sugerentes de SAOS, ya que éste podría presentarse como factor causal o como condición contribuyente o agravante de dichas patologías.

Cabe aclarar las numerosas debilidades de nuestro estudio, dado su carácter observacional y descriptivo, con una población muestral escasa que incluye gran número de pacientes jóvenes. 


\section{Conclusión}

Destacamos la relación, ya descrita en la literatura, entre los factores de RCV y la AOS que, si bien no fue demostrable en todos los casos, fue notoria el los de HTA, diabetes y obesidad, condiciones altamente prevalentes en nuestra sociedad. Es imperativo considerar al SAOS como un factor de RCV modificable, involucrándonos en su manejo con el objetivo de mejorar la calidad de vida, reducir el riesgo de las consecuencias crónicas y disminuir los costos que supone en el sistema de la salud.

\section{Bibliografía}

1. Kapur, V. K., Auckley, D. H., Chowdhuri, S., Kuhlmann, D. C., Mehra, R., Ramar, K., et al. Clinical practice guideline for diagnostic testing for adult obstructive sleep apnea: an American Academy of Sleep Medicine clinical practice guideline. J Clin Sleep Med, 2017; 13(3), 479-504.

2. Barashi, N. S., Ruiz, R. E., Marín, L., Ruiz, P., Amado, S., Ruiz, Á. J., \& Hidalgo, P. Síndrome de apnea/hipopnea obstructiva del sueño y su asociación con las enfermedades cardiovasculares. Rev Col Cardiololgía, 2015; 22(2), 81-87.

3. Marin, J. M., Agusti, A., Villar, I., Forner, M., Nieto, D., Carrizo, S. J. et al. Association between treated and untreated obstructive sleep apnea and risk of hypertension. Jama, 2012; 307(20), 2169- 2176.

4. Punjabi, N. M., Caffo, B. S., Goodwin, J. L., Gottlieb, D. J., Newman, A. B., O'Connor, et al. Sleepdisordered breathing and mortality: a prospective cohort study. PLoS medicine, 2009; 6(8), e1000132.

5. Zamora, E., Casanova, A., Chao, P., Domingo, M. J., Gutiérrez, B., Moral, M., et al. Síndrome de apnea obstructiva del sueño y factores de riesgo cardiovascular. Rev Patol Respir, 2005; 8(2), 162- 165.

6. Drager, L. F., Bortolotto, L. A., Lorenzi, M. C., Figueiredo, A. C., Krieger, E. M., \& Lorenzi-Filho, G. Early signs of atherosclerosis in obstructive sleep apnea. AJRCCM, 2005; 172(5), 613-618.

7. Patt, B. T., Jarjoura, D., Haddad, D. N., Sen, C. K., Roy, S., Flavahan, N. A., et al. Endothelial dysfunction in the microcirculation of patients with obstructive sleep apnea. AJRCCM 2010; 182(12), 15401545 .
8. Peppard, P. E., Young, T., Palta, M., \& Skatrud, J. Prospective study of the association between sleep-disordered breathing and hypertension. NEYM, 2000; 342(19), 1378-1384.

9. Peker, Y., Hedner, J., Norum, J., Kraiczi, H., \& Carlson, J. Increased incidence of cardiovascular disease in middle-aged men with obstructive sleep apnea: a 7-year followup. AJRCCM, 2002; 166(2), 159-165.

10. Lam, J. C. M., Mak, J. C. W., \& Ip, M. S. M. Obesity, obstructive sleep apnea and metabolic syndrome. Respirology, 2012; 17(2), 223-236.

11. Gozal, D., \& Kheirandish-Gozal, L. Cardiovascular morbidity in obstructive sleep apnea: oxidative stress, inflammation, and much more. AJRCCM, 2008; 177(4), 369375.

12. Bhattacharjee, R., Kheirandish-Gozal, L., Pillar, G., \& Gozal, D. Cardiovascular complications of obstructive sleep apnea syndrome: evidence from children. Progress in cardiovascular diseases, 2009; 51(5), 416433.

13. Drager L.F, Jun, J.C., Polotsky, V.Y. Consecuencias metabólicas de la hipoxia intermitente: relevancia para la apnea obstructiva del sueño. Best Pract Res Clin Endocrinol Metab, 2010; 24(5): 843-851.

14. Kaditis, A. G., Alexopoulos, E. I., Kalampouka, E., Kostadima, E., Angelopoulos, N., Germenis, A, et al. Morning levels of fibrinogen in children with sleep-disordered breathing. ERJournals, 2004; 24(5), 790-797

15. Moreno, G. M. Definición y clasificación de la obesidad. RMCLC, 2012; 23(2), 124-128.

16. Rubio, M. A., Moreno, C., \& Cabrerizo, L. Guías para el tratamiento de las dislipemias en el adulto: Adult Treatment Panel III (ATPIII). Endocrinología y Nutrición, 2014; 51(5), 254-265.

17. Benzadón, M., Forti, L., \& Sinay, I. Actualización en el diagnóstico de la diabetes. Medicina (Buenos Aires), 2014; 74(1), 64-68.

18. Mancia, G., Fagard, R., Narkiewicz, K., Redon, J., Zanchetti, A., \& Böhm, M. Guía de práctica clínica de la ESH/ESC 2013 para el manejo de la hipertensión arterial. Rev Esp Cardiol, 2013; 66(10).

19. Stone A., Neil J. "2013 ACC/AHA guideline on the treatment of blood cholesterol to reduce 

apnea obstructiva del sueño.

atherosclerotic cardiovascular risk in adults." Circulation, 2014; 25 (2), 1-45.

20. Prevention of Cardiovascular Disease. Guidelines for assessment and management of cardiovascular risk. World Health Organization, 2007. ISBN 9789241547178.

21. McNicholas, W.T. Diagnosis of obstructive sleep apnea in adults. Proc Am Thorac Soc, 2008; 5, 154-160.

22. Gunduz, C., Kacmaz Basoglu, O., Hedner, J., Bonsignore, M.R., Hein, H., Staats, R., et al. Hyperlipidaemia prevalence and cholesterol control in obstructive sleep apnoea: Data from the European sleep apnea database (ESADA). J Intern Med. 2019.

23. Loke, Y., William, J., Brown, L., Kwok, C.S., Niruban, A. \& Myint, P.K. Association of Obstructive Sleep Apnea with Risk of Serious Cardiovascular Events A Systematic Review and Meta-Analysis Circ Cardiovasc Qual Outcomes, 2012; 5, 720-728.

24. Randerath, W., Bonsignore, M.R., S. Obstructive sleep apnea in acute coronary syndrome. European Respiratory Review, 2019; 28, 180114.

25. Ryan, S., Arnaud, C., Fitzpatrick, S.F., Gaucher, J., Tamisier, R. \& Pépin, J.L. Adipose tissue as a key player in obstructive sleep apnea. European Respiratory Review, 2019; 28, 190006.

26. Diaz, A.A., Espeche, W., March, C., Flores, R., Parodi, R., et al. Prevalencia del síndrome metabólico en Argentina en los últimos 25 años: revisión sistemática de estudioso observacionales poblacionales. Elsevier: Hipertension y Riesgo Vascular; 2018; 35 (2), 64-69.

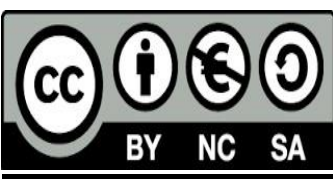

